\title{
Sarcoidose laríngea: Revisão de literatura
}

\section{Laryngeal sarcoidosis: Literature review}

\section{Bruno Teixeira de Moraes' ${ }^{,}$Luciano Rodrigues Neves², Osíris de Oliveira Camponês do Brasil ${ }^{3}$, José Eduardo de Sá Pedroso ${ }^{4}$, Jose Elson Santiago de Melo Junior.}

1) Otorrinolaringologista. Mestrando em Medicina pelo Departamento de Otorrinolaringologia e Cirurgia de Cabeça e Pescoço da Universidade Federal de São Paulo (UNIFESP-EPM).

2) Otorrinolaringologista. Doutorando em Medicina pelo Departamento de Otorrinolaringologia e Cirurgia de Cabeça e Pescoço da Universidade Federal de São Paulo (UNIFESP-EPM)

3) Otorrinolaringologista. Professor Doutor do Departamento de Otorrinolaringologia e Cirurgia de Cabeça e Pescoço da Universidade Federal de São Paulo (UNIFESPEPM).

4) Otorrinolaringologista. Assistente Doutor do Departamento de Otorrinolaringologia e Cirurgia de Cabeça e Pescoço da Universidade Federal de São Paulo (UNIFESPEPM).

5) Médico. Especializando em Otorrinolaringologia da Universidade Federal de São Paulo (UNIFESP-EPM).

Instituição: Setor de Laringologia e Voz da Disciplina de Otorrinolaringologia da Universidade Federal de São Paulo - Escola Paulista de Medicina (UNIFESP-EPM). São Paulo / SP - Brasil.

Endereço para correspondência: Bruno Teixeira de Moraes - Rua Pedro de Toledo, 957 - Vila Clementino - São Paulo / SP - Brasil - CEP: 04039-032 - Telefone (+55 11) 5573-2740 - E-mail: moraesbruno.orl@ hotmail.com

Artigo recebido em 23 de Janeiro de 2010. Artigo aprovado em 24 de Abril de 2010.

\section{RESUMO}

Introdução: A sarcoidose laríngea é uma doença granulomatosa que representa um desafio em relação ao diagnóstico e o tratamento.

Método: Nesta revisão são abordados todos os aspectos da doença, incluindo etiologia, manifestações clínicas em adultos e crianças, diagnóstico e tratamento, a partir da base de dados Medline, Lilacs e Scielo, incluindo-se artigos de língua inglesa e portuguesa publicados no período de 1973 a 2008. Comentários Finais: Apesar dos avanços ocorridos na investigação e tratamento da sarcoidose, é uma doença que ainda se mantém sem etiologia e fisiopatologia definidas, constituindo um diagnóstico de exclusão pela ausência de exames específicos e por sua provável origem multifatorial. O acometimento laríngeo, apesar de raro, é suspeitado principalmente diante de história prévia de sarcoidose sistêmica, e a pesquisa dos diagnósticos diferenciais deve ser extensa para não passarem desapercebidas doenças graves como neoplasias malignas.

Palavras-chave: doenças da laringe, sarcoidose, laringe, tomografia por emissão de pósitrons.

\section{SUMMARY}

Introduction: Laryngeal sarcoidosis is a granulomatous disease representing a challenge as regards diagnosis and treatment. Method: In this review, all aspects of the disease are involved, including etiology, clinical manifestations in adults and children, diagnosis, and treatment, by searching through Medline, Lilacs and Scielo databases, as well as English and Portuguese articles published from 1973 to 2008.

Final Comments: Despite the breakthroughs in its diagnosis and treatment, sarcoidosis is a disease that remains without a clear etiology and physiopathology, becoming an exclusion diagnosis due to both the lack of precise exams and its likely multifactor background. Laryngeal impairment, however infrequent, is a suspicion mainly when there is a precedent history of systemic sarcoidosis, and the research for differentiated diagnosis must be wide-ranging so as to prevent severe diseases like malign neoplasia from being unnoticed. Keywords: laryngeal disease, sarcoidosis, larynx, positron emission tomography. 


\section{INTRODUÇÃO}

As granulomatoses são doenças sistêmicas que fazem parte do diagnóstico diferencial de inúmeras afecções do trato respiratório, de um lado pela predileção por esse sistema e por outro pelo polimorfismo de suas manifestações clínicas. Fazem parte deste grupo a sarcoidose, doença inflamatória crônica de etiologia indefinida, que na maioria dos casos acomete os pulmões, as pleuras viscerais e parietais, linfonodos hilares e mediastinais. Na região da cabeça e do pescoço pode causar linfadenopatia, paralisia facial, inchaço parotídeo, lesão ocular, nasal e laríngea, mas raramente de forma isolada.

A sarcoidose laríngea é uma doença subdiagnosticada quer seja por não haver testes específicos para sua detecção, constituindo diagnóstico de exclusão, como pela multiplicidade de critérios a serem analisados para que a suspeita diagnóstica se confirme (1). O quadro clínico pode variar desde a forma assintomática a casos graves, principalmente quando há obstrução de vias aéreas. Observam-se diferenças na apresentação da doença de acordo coma idade, como uma menor frequência do acometimento laríngeo isoladoesintomatologia mais evidentena faixa etária pediátrica.

Novas tecnologias têm sido aplicadas no diagnóstico, como o PET/CT (tomografia por emissão de pósitrons) que tem ampla utilização em neoplasias malignas, mas como não é um exame tumor-específico, pode ser utilizado na detecção de condições benignas como sarcoidose.

O objetivo desta revisão é atualizar os dados sobre sarcoidose, desde etiologia até tratamento, com ênfase no acometimento laríngeo que é pouco descrito na literatura e abordar uma investigação criteriosa no seu diagnóstico diferencial.

\section{MÉTODO}

Esta revisão foi realizada através de busca dos descritores de assunto "sarcoidosis", "laryngeal diseases" e "larynx" nas bases de dados Medline, Lilacs e Scielo, incluindo-se artigos de língua inglesa e portuguesa publicados no período de 1973 a 2008. A partir da seleção primária foram acrescentados novos trabalhos científicos relevantes encontrados nas referências bibliográficas.

\section{REVISÃO DE LITERATURA}

\section{Epidemiologia}

A sarcoidose incide em pessoas de todas as idades, sexos e raças, contudo com algumas diferenças na forma de apresentação e frequência por prováveis fatores genéticos e ambientais envolvidos. Tipicamente ocorre em adultos jovens dos 20 aos 40 anos, e há uma predominância discreta em mulheres. Nos EUA, identificou-se maior prevalência entre negros (35,5/100.000 habitantes) em relação à população branca (10,9/100.000). Já no continente africano nota-se também maior prevalência em negros, contudo com coeficientes menores. Nos países escandinavos, encontram-se prevalências mais elevadas, em torno de 64/100.000 na Suécia e 26,7/100.000 na Noruega (2). No Brasil, a prevalência na população geral foi estimada em torno de 10/100.000 habitantes (3). O envolvimento da laringe ocorre em torno de 0,5 a $8,3 \%$ dos pacientes, e sua presença isolada neste órgão é ainda mais rara $(4,5)$.

\section{Etiologia}

Vários fatores etiológicos têm sido associados como prováveis causas da sarcoidose, aventando-se a possibilidade de que agentes ambientais infecciosos ou não desencadeiem uma cascata de eventos imunológicos e inflamatórios, com transformação final de macrófagos em células gigantes eepitelioides, as quais formamblocos de granuloma e caracterizam a doença em pessoas que são geneticamente susceptíveis $(6,7)$.

A sarcoidose surge frequentemente em indivíduos expostos a ambientes nos quais outras granulomatoses comprovadamente ocorrem, em particular, exposição ocupacional a inseticidas, emprego na agricultura, criação de pássaros e ambientes úmidos e mofados, tipicamente associados com exposição a bioaerossóis (8).

A susceptibilidade genética é demonstrada pela evidência de maior prevalência na forma familiar e correlação de alguns genótipos com a gravidade e cronicidade da doença (9.10), além da demonstração de associação com alguns alelos HLA (DRB1 e DQB1) (11,12).

Pela semelhança do quadro clínico e histológico com doenças causadas por fungos e micobactérias, possibilidade transmissão em pacientes transplantados, aumento de anticorpos e detecção nos tecidos de alguns agentes como Propionibacteracnes sugere-se uma causa infecciosa para sarcoidose $(13,14)$.

\section{Manifestações Clínicas}

Identificam-se três padrões clínicos de pacientes diagnosticados como portadores de sarcoidose: sem sintomas, sintomas constitucionais inespecíficos e sintomas 
órgão- específicos (1). Os pacientes assintomáticos têm investigação iniciada a partir de alterações detectadas em radiografia de tórax de rotina, e perfazem em torno de 30 a $50 \%$ do total $(15,16)$. Os pacientes sintomáticos apresentam queixas inespecíficas tais como fadiga, febre e perda de peso; ou associadas a sintomas órgão-específicos. Em relação à laringe os sinais e sintomas mais comuns são globo faríngeo, dispneia, disfonia, disfagia e tosse seca. Casos mais graves podem evoluir com estridor e obstrução de via aérea, às vezes com necessidade de traqueostomia $(1,17)$.

Os pacientes com sarcoidose laríngea não necessariamente apresentam a forma sistêmica; na revisão de Benjamin et al (18), de cinco pacientes com lesões laríngeas, apenas um apresentava doença generalizada; nos relatos de Neel and Mcdonald (4), entre treze pacientes com lesões laríngeas, sete apresentavam acometimento de outros órgãos.

Os achados à laringoscopia se apresentam como mucosa edematosa, sobre-elevada e pálida nos seguintes sítios em ordem decrescente de frequência: epiglote, aritenoides, pregas ariepiglóticas e pregas vestibulares (19). Esse acometimento principal da supraglote ocorre provavelmente pela grande quantidade de vasos linfáticos nessa região que se encontram alargados com substituição da arquitetura por depósitos sarcoides ou focos subcutâneos da doença (Figura 1) (20). Pode, mais raramente, envolver subglote e em $24 \%$ dos casos as pregas vocais, inclusive causando imobilidade das mesmas, seja por comprometimento do nervo vago ou fixação destas por infiltração sarcoide na articulação cricoaritenoidea, diagnóstico por vezes difícil de ser realizado através de eletromiografia $(21,22)$.

\section{Sarcoidose Pediátrica}

Esta é uma doença incomum na faixa etária pediátrica, com incidência em torno de 1,02/100.000 (23). Geralmente se apresenta de forma mais sintomática do que em adultos, provavelmente porque a doença é mais detectada em adultos assintomáticos através de radiografia de tórax de rotina, que geralmente não é realizada em crianças (24). Os sintomas mais comuns são letargia e fadiga, mas também são frequentes tosse, febre e perda de peso (25).

Sua apresentação extrapulmonar é mais frequente do que em adultos, contudo o acometimento laríngeo isolado é mais raro, como relatado por KenNy et al. (26) O envolvimento laríngeo ocorre tanto na faixa pré-puberal quanto pós-puberal, e são frequentes dispneia, tosse e rouquidão (27).

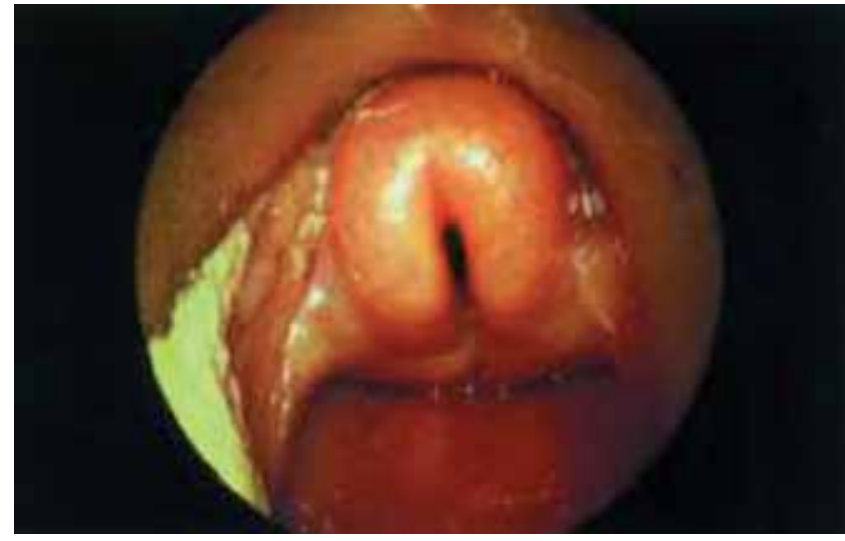

Figura 1. Sarcoidose Laríngea - Imagem cedida pelo Dr. Enric Massana.

\section{Diagnóstico}

Para o diagnóstico de sarcoidose deve-se preencher 3 critérios: achados clínico-radiológicos compatíveis, amostra histológica representada por granulomas não caseosos e exclusão de outras granulomatoses ou doenças com achados semelhantes.

As imagens radiológicas do tórax geralmente mostram linfadenopatia hilar e infiltrado pulmonar (6). As imagens da laringe são mais inespecíficas, dentre elas o raios-X cervical em perfil que pode mostrar aumento da epiglote, semelhante ao "sinal do polegar" da epiglotite aguda, ou a tomografia computadorizada que apresenta massa com densidade de partes moles na topografia acometida, geralmente supraglote $(18,28)$.

Outro exame utilizado mais recentemente é o FDG PET (tomografia por emissão de pósitrons) que tem identificado aumento na captação do flúor-deoxi-glicose não só em lesões malignas, mas também em lesões granulomatosas como sarcoidose $(29,30)$.

Braun et al. (31) demonstrou uma sensibilidade 85\% do PET/CT em detectar sítios ativos de sarcoidose laringofaríngea comprovados por biópsia. Além disso, pode ser um método útil para alertar a presença de envolvimento laríngeo em pacientes com historia prévia de sarcoidose que não tenham sintomas característicos (29).

Evidência de lesão em laringe associada à amostra histológica de outro sítio (por exemplo pulmonar) compatível com sarcoidose, não são suficientes para determinar acometimento laríngeo pela sarcoidose, pois na literatura são descritos casos de sarcoidose pulmonar associada a carcinoma laríngeo (32). Portanto, para definir diagnóstico de sarcoidose laríngea deve-se proceder à biópsia da lesão 
Tabela I. Diagnóstico diferencial da sarcoidose.

\begin{tabular}{lccc}
\hline Infecciosas & Inalantes & Indeterminadas & Miscelânea \\
\hline $\begin{array}{l}\text { Tuberculose } \\
\text { Hanseníase }\end{array}$ & Silicose & Wegener & Hipotireoidismo \\
Sífilis & & \\
Paracoccidioidomicose & Asbestose & & \\
Histoplasmose & & Amiloidose & Carcinomaescamocelular \\
Rinoescleroma & Berilose & & \\
Actinomicose & & & \\
\hline
\end{tabular}

nessa topografia, cujo resultado apresenta-se como granuloma epitelioide não caseoso $(1,32)$.

Para exclusão de outras doenças que fazem farte do diagnóstico diferencial, deve-se solicitar pesquisa de fungos e bacilos álcool-ácido resistentes, além de cultura para bactérias e fungos a partir do espécime biopsiado; pesquisa de sífilis (VDRL), granulomatose de Wegener (cANCA), tuberculose (PPD), e sorologia para histoplasmose e paracoccidioidomicose $(1,18)$.

\section{Diagnóstico Diferencial}

Como a sarcoidose é um diagnóstico de exclusão (1, 21), deve-se utilizar todos os métodos complementares para investigação de doenças que têm manifestações semelhantes nos parâmetros clínicos, radiológicos, e principalmente histológicos (Tabela 1).

\section{Tratamento}

O curso clínico da sarcoidose é muito variável: 60 a 70\% dos pacientes apresentam remissão espontânea, 1020\% apresentam sequelas permanentes e a mortalidade é de 1 a $5 \%$, geralmente por complicações pulmonares, cardíacas e neurológicas (1). O tratamento de primeira escolha é a corticoterapia sistêmica, geralmente a droga utilizada é a prednisona na dose 40 a $60 \mathrm{mg} / \mathrm{dia}$ em adultos e $1 \mathrm{mg} / \mathrm{kg} /$ dia em crianças, durante um período total de 6 a 12 meses $(34,35)$. Nos casos refratários ou com finalidade de diminuir efeitos colaterais dos corticoides, podem ser usados com boa eficácia agentes citotóxicos como metotrexato e azatioprina, e apenas em casos resistentes utilizam-se drogas mais tóxicas como ciclofosfamida e clorambucil $(36,37)$.

Quando não há uma melhora satisfatória de lesões laríngeas com tratamento sistêmico e o paciente apresenta-se sintomático, uma opção é a injeção intralesional de corticoide (triancinolona ou metilprednisolona), que atingirá altas concentrações locais com efeitos colaterais reduzi- dos (26). Os resultados são variáveis, mas em alguns casos de obstrução de vias aéreas alcança-se a decanulação da traqueostomia com essa terapêutica (27). Quando há iminência de insuficiência respiratória por obstrução alta, deve ser realizada a traqueostomia precocemente, e se não houver resposta a corticoterapia injetável opta-se por ressecção de lesões obstrutivas, na maioria das vezes, ressecção parcial da epiglote através de laser de CO2 ou instrumentos frios (26). Também é descrito tratamento radioterápico para essas lesões, com a desvantagem de possível efeito carcinogênico local (38).

\section{COMENTÁRIOS FINAIS}

Nesta revisão são mostrados os avanços na investigação e tratamento da sarcoidose, porém é uma doença que ainda se mantém sem etiologia e fisiopatologia definidas, constituindo um diagnóstico de exclusão pela ausência de exames específicos e por sua provável origem multifatorial. O acometimento laríngeo, apesar de raro, é suspeitado principalmente diante de história prévia de sarcoidose sistêmica, e a pesquisa dos diagnósticos diferenciais deve ser extensa para não passarem desapercebidas doenças graves como neoplasias malignas.

\section{REFERÊNCIAS BIBLIOGRÁFICAS}

1. Schwartzbauer HR, Tami TA. Ear, nose and throat manifestations of sarcoidosis. Otolaryngol Clin N Am. 2003, 36:673-84.

2. James DG, Hosoda Y. Epidemiology in sarcoidosis and other granulomatous disorders. New York: Marcel Dekker; 1994. (Lung biology in health and disease).

3. Bethlem NM. Epidemiology of sarcoidosis in Brazil. Sarcoidosis. 1985, 2:162.

4. Neel HB III, McDonald TJ. Laryngeal sarcoidosis: report of 13 patients. Ann Otol Rhinol Laryngol. 1982, 91(4 Pt 1):359-62. 
5. Fortune S, Courey MS. Isolated laryngeal sarcoidosis. Otolaryngol Head Neck Surgery. 1998, 118(6):868-70.

6. Weinberger SE. Sarcoidosis. In: Goldman: Cecil medicine. Philadelphia: Elsevier Saunders; 2007. chapter 95.

7. Shigehara K, Shijubo N, Ohmichi M, et al. Enhanced mRNA expression of Th1 cytokines and IL-12 in active pulmonary sarcoidosis. Sarcoidosis Vasc Diffuse Lung Dis. 2000, 17(2):151-7.

8. Newman LS, Rose CS, Bresnitz EA, Rossman MD, Barnard J, Frederick M, et al. A case control etiologic study of sarcoidosis: environmental and occupational risk factors. Am J Respir Crit Care Med. 2004, 170:1324-1330.

9. Brennan NJ, Crean P, Long JP, et al. High prevalence of familial sarcoidosis in an Irish population. Thorax. 1984, 39(1):14-8.

10. Abe S, Yamaguchi E, Makimura S, et al. Association of HLA-DR with sarcoidosis. Correlation with clinical course. Chest. 1987, 92(3):488-90.

11. Rossman MD, Thompson B, Frederick M, et al. HLADRB1 1101: a significant risk factor for sarcoidosis in blacks and whites. Am J Hum Genet. 2003, 73:720-35.

12. Iannuzzi MC, Maliarik MJ, Poisson LM, Rybicki BA. Sarcoidosis susceptibility and resistance HLA-DQB1 alleles in African Americans. Am J Respir Crit Care Med. 2003, 167:1225-31.

13. Heyll A, Meckenstock G, AulC, et al. Possible transmission of sarcoidosis via allogeneic bone marrow transplantation. Bone Marrow Transplant. 1994, 14(1):161-4.

14. Moller DR, Chen ES. What causes sarcoidosis? Current Opinion in Pulmonary Medicine. 2002, 8:429-434.

15. Siltzbach LE, James DG, Neville E, et al. Course and prognosis of sarcoidosis around the world. Am J Med. 1974, 57(6):847-52.

16. Winterbauer RH, Belic N, Moores KD. Clinical interpretation of bilateral hilar adenopathy. Ann Intern Med. 1973, 78(1):65-71.

17. Vaz FM, Samuel D. Postcricoid sarcoid mimicking a malignancy: A lesson to remember. Otolaryngol Head Neck Surg. 2000, 123:150.

18. Benjamin B, Dalton C, Croxson G. Laryngoscopic Diagnosis Of Laryngeal Sarcoid. Ann Otol Rhinol Laryngol. 1995, 104:529-31.
19. Dean CM, Sataloff RT, Hawkshaw MJ, Pribikin E. Laryngeal sarcoidosis. J Voice. 2002, 16(2):283-8.

20. Hernández PE, Casas AA, Massana EF, et al. Sarcoidosis Laríngea Aislada. Acta Otorrinolaringol Esp. 2003, 54:117-20.

21. Caporrino Neto J, Cervantes O, Jotz GP, Abrahão M. Doenças granulomatosas da Laringe. Acta Awho. 1998, 17(1):6-10.

22. Bower JS, BelenJE, WegJG, Dantzer DR. Manifestations and treatment of laryngeal sarcoidosis. Am Rev Respir Dis. 1980, 122:325-32.

23. Hoffmann AL, Milman N, Byg KE. Childhood sarcoidosis in Denmark 1979-1994: incidence, clinical features and laboratory results at presentation in 48 children. Acta Paediatr. 2004, 93:30-6.

24. Kendig EL. The clinical picture of sarcoidosis in children. Pediatrics. 1974, 54: 289-92.

25. Kendig EL, Brummer DL. The prognosis of sarcoidosis in children. Chest. 1976, 70:351-353.

26. Kenny TJ, Werkhaven J, Netterville JL. Sarcoidosis of the Pediatric Larynx. Arch Otolaryngol Head Neck Surg. 2000, 126:536-39.

27. Rybak L, Falconeer R. Pediatric Laryngeal Sarcoidosis. Ann Otol Rhinol Laryngol. 1987, 96:670-73.

28. Palacios E, Smith A, Gupta N. Laryngeal sarcoidosis. Ear, Nose and Throat Journal. 2008, 252-53.

29. Kaira K, Ishizuka T, Yanagitani N, Sunaga N, Hisada T, Mori M. Laryngeal Sarcoidosis Detected by FDG Positron Emission Tomography. Clin Nucl Med. 2008, 33:878-79.

30. Avram AM, Mackie GC, Schneider BJ, et al. Differentiation between carcinoid and sarcoid with F-18 FDG PET and In111 pentetreotide. Clin Nucl Med. 2006, 31:197-200.

31. Braun JJ, Imperiale A, Schultz P, Molard A, Charpiot A, Gentine A. Pharyngolaryngeal sarcoidosis: Report of 12 cases. Otolaryngology-Head and Neck Surgery. 2008, 139:463-65.

32. Gal AA, Koss MN. The pathology of sarcoidosis. Current Opinion in Pulmonary Medicine. 2002, 8:445-451.

33. Iannuzzi MC, Rybicki BA, Teirstein AS. Sarcoidosis. N Engl J Med. 2007, 357:2153-65.

34. Martinez JAB. Doenças intersticiais pulmonares. Medicina. 1998, 31:247-256. 
35. Newman LS, Rose CS, Maier LA. Sarcoidosis. N Engl J Med. 1997, 336(17):1224-1234.

36. Baughman RP, Lower EE. A clinical approach to the use of methotrexate for sarcoidosis. Thorax. 1999, 54(8):7426.
37. Lewis SJ, Ainslie GM, Bateman ED. Efficacy of azathioprine as second-line treatment in pulmonary sarcoidosis. Sarcoidosis Vasc Diffuse Lung Dis. 1999, 16(1):87-92.

38. Carasso B. Sarcoidosis of the larynx causing airway obstruction. Chest. 1974, 65(6):693-5. 